Supplement of Geosci. Model Dev., 12, 1909-1963, 2019

https://doi.org/10.5194/gmd-12-1909-2019-supplement

(C) Author(s) 2019. This work is distributed under

the Creative Commons Attribution 4.0 License.

(c) (1)

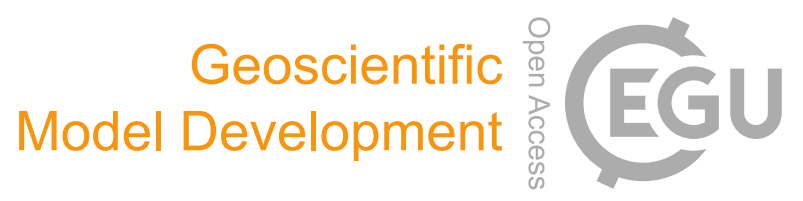

Supplement of

\title{
The Met Office Unified Model Global Atmosphere 7.0/7.1 and JULES Global Land 7.0 configurations
}

David Walters et al.

Correspondence to: David Walters (david.walters@metoffice.gov.uk)

The copyright of individual parts of the supplement might differ from the CC BY 4.0 License. 


\section{Contents}

1 Introduction 1

$\begin{array}{lll}2 & \text { GA7.0 settings that vary with global horizontal resolution } & 2\end{array}$

3 GA7.0 settings that vary with vertical resolution 2

3.1 UM settings to change with level set . . . . . . . . . . . . . . 2

3.2 Details of vertical level sets . . . . . . . . . . . . . . . . . . . 2

4 Namelist differences between GA7.0 and GA7.1 5

5 Settings that may vary with system/application 5

\section{Introduction}

This supplementary material to the main paper is designed to help users of GA7.0/7.1 and GL7.0 in configuring their systems to correctly implement the new configurations. 


\section{GA7.0 settings that vary with global horizontal resolution}

Table 1 lists the Unified Model (UM) settings (as set in the Rose suite applications - see the Rose documentation at http://metomi.github.io/rose/doc/rose.html) that should be changed when changing horizontal resolution. The settings listed here are valid for UM code base vn10.6. The resolutions supported span from $\mathrm{N} 96(\approx 135 \mathrm{~km}$ in the mid-latitudes $)$ to $\mathrm{N} 1280(\approx 10 \mathrm{~km}$ in the mid-latitudes).

\begin{tabular}{|c|c|c|c|c|c|c|c|c|}
\hline $\begin{array}{c}\text { namelist } \\
\text { entry }\end{array}$ & N96 & N216 & N320 & N400 & N512 & N768 & N1024 & N1280 \\
\hline \multicolumn{9}{|l|}{ [namelist:nlsizes] } \\
\hline global_row_length & 192 & 432 & 640 & 800 & 1024 & 1536 & 2048 & 1560 \\
\hline global_rows & 144 & 324 & 480 & 600 & 768 & 1152 & 1536 & 1280 \\
\hline \multicolumn{9}{|l|}{ [namelist:nlst_mpp] } \\
\hline extended_halo_size_ew & 4 & 4 & 4 & 4 & 5 & 5 & 5 & 5 \\
\hline $\begin{array}{l}\text { extended_halo_size_ns } \\
\text { [namelist:nlstcgen] }\end{array}$ & 5 & 7 & 7 & 8 & 8 & 8 & 10 & 10 \\
\hline $\begin{array}{l}\text { steps_per_periodim } \\
\text { [namelist:run_dust] }\end{array}$ & 72 & 96 & 120 & 120 & 144 & 192 & 288 & 360 \\
\hline $\begin{array}{l}\text { us_am } \\
\text { [namelist:run_gwd] }\end{array}$ & 1.45 & 1.40 & 1.40 & 1.40 & 1.40 & 1.40 & 1.40 & 1.40 \\
\hline ussp_launch_factor & 1.3 & 1.2 & 1.2 & 1.2 & 1.2 & 1.2 & 1.2 & 1.2 \\
\hline
\end{tabular}

Table 1: GA7.0 settings that vary with global horizontal resolution.

\section{GA7.0 settings that vary with vertical resolution}

\subsection{UM settings to change with level set}

Table 2 lists the UM settings (as set in the Rose suite applications) that should be changed when changing vertical resolution. The settings listed here are valid for UM code base vn10.6. Note that as discussed in the main paper, GA7.0 systems should only use either $L 85\left(50_{t}, 35_{s}\right)_{85}$, $\mathrm{L} 70\left(50_{\mathrm{t}}, 2 \mathrm{O}_{\mathrm{s}}\right)_{80}$, or L63 $\left(50_{\mathrm{t}}, 13_{\mathrm{s}}\right)_{40}$ level sets.

\subsection{Details of vertical level sets}

In the vertical, the UM uses the terrain-following height coordinate $\eta$, which is normalised to be $\eta=0$ at the lower boundary, and $\eta=1$ at a height $z_{T}$, the height of the fixed model lid. In between, the height above mean sea level at any given point, $z$, is defined by

$$
z= \begin{cases}\eta z_{T}+h\left(1-\frac{\eta}{\eta_{I}}\right)^{2}, & 0 \leq \eta \leq \eta_{I} \\ \eta z_{T}, & \eta_{I} \leq \eta \leq 1,\end{cases}
$$

where $h$ is the height of the model orography above the earth's mean radius and $\eta_{I}$ is the level at and above which the levels are flat.

The namelists below detail the level sets used with GA7.0. In these namelists, the variable $z_{-}$top_of model $=z_{T}$ (in metres), eta_theta is the array of $\eta$ values for the levels on which the prognostic potential temperature $(\theta)$ is held including the surface, eta_rho is the array of $\eta$ values for the levels on which the prognostic density $(\rho)$ is held, and first_constant_r_rho_level is the $\rho$-level at which $\eta=\eta_{I}$. 


\begin{tabular}{lrrr}
\hline $\begin{array}{l}\text { namelist } \\
\quad \text { entry }\end{array}$ & $\mathrm{L} 85\left(50_{\mathrm{t}}, 35_{\mathrm{s}}\right)_{85}$ & $\mathrm{~L} 70\left(50_{\mathrm{t}}, 20_{\mathrm{s}}\right)_{80}$ & $\mathrm{L63}\left(50_{\mathrm{t}}, 13_{\mathrm{s}}\right)_{40}$ \\
\hline $\begin{array}{l}\text { [namelist:domain("All model level STASH domains") } \\
\quad \text { levt }\end{array}$ & 85 & 70 & 63 \\
$\begin{array}{l}\text { [namelist:nlsizes] } \\
\quad \text { cloud_levels }\end{array}$ & 85 & 70 & 63 \\
$\quad$ model_levels & 85 & 70 & 63 \\
$\quad$ ozone_levels & 85 & 70 & 63 \\
$\begin{array}{l}\text { [namelist:run_cloud] } \\
\text { rhcrit(18:) }\end{array}$ & $68 * 0.800$ & $53^{*} 0.800$ & $46^{*} 0.800$ \\
[namelist:run_dyn] & & & \\
$\quad$ eta_s & 0.5 & 0.5 & 0.75 \\
\hline
\end{tabular}

Table 2: GA7.0 settings that vary with atmospheric vertical resolution. Note that whilst the PC2 cloud scheme now uses a diagnostic formulation for rhcrit $\left(R H_{\text {crit }}\right)$ as described in section 3.4 of the main paper, a prescribed $R H_{\text {crit }}$ profile is still in the microphysics scheme to determine the in-cloud relative humidity.

Level set $\mathbf{L} 85\left(50_{\mathrm{t}}, 35_{\mathrm{s}}\right)_{85}$

\begin{tabular}{|c|c|c|c|c|}
\hline \multicolumn{5}{|l|}{ \&VERTLEVS } \\
\hline$z_{-}$top_of_model & 85000.00 & & & \\
\hline \multicolumn{5}{|c|}{$\begin{array}{l}\text { first_constant_r_rho-level= } 51 \text {, } \\
\text { eta_theta= }\end{array}$} \\
\hline $0.0000000 \mathrm{E}+00$ & $0.2352941 \mathrm{E}-03$ & $0.6274510 \mathrm{E}-03$ & $0.1176471 \mathrm{E}-02$ & $0.1882353 \mathrm{E}-02$, \\
\hline $0.2745098 \mathrm{E}-02$, & $0.3764706 \mathrm{E}-02$ & $0.4941176 \mathrm{E}-02$ & $0.6274510 \mathrm{E}-02$ & $0.7764705 \mathrm{E}-02$, \\
\hline $0.9411764 \mathrm{E}-02$, & $0.1121569 \mathrm{E}-01$ & $0.1317647 \mathrm{E}-01$ & $0.1529412 \mathrm{E}-01$ & $0.1756863 \mathrm{E}-01$, \\
\hline $0.2000000 \mathrm{E}-01$, & $0.2258823 \mathrm{E}-01$ & $0.2533333 \mathrm{E}-01$ & $0.2823529 \mathrm{E}-01$ & $0.3129411 \mathrm{E}-01$, \\
\hline $0.3450980 \mathrm{E}-01$, & $0.3788235 \mathrm{E}-01$ & $0.4141176 \mathrm{E}-01$ & $0.4509804 \mathrm{E}-01$ & $0.4894118 \mathrm{E}-01$, \\
\hline $0.5294117 \mathrm{E}-01$, & $0.5709804 \mathrm{E}-01$ & $0.6141176 \mathrm{E}-01$ & $0.6588235 \mathrm{E}-01$ & $0.7050980 \mathrm{E}-01$, \\
\hline $0.7529411 \mathrm{E}-01$, & $0.8023529 \mathrm{E}-01$ & $0.8533333 \mathrm{E}-01$ & $0.9058823 \mathrm{E}-01$ & $0.9600001 \mathrm{E}-01$, \\
\hline $0.1015687 \mathrm{E}+00$ & $0.1072942 \mathrm{E}+00$ & $0.1131767 \mathrm{E}+00$ & $0.1192161 \mathrm{E}+00$ & $0.1254127 \mathrm{E}+00$, \\
\hline $0.1317666 \mathrm{E}+00$ & $0.1382781 \mathrm{E}+00$ & $0.1449476 \mathrm{E}+00$ & $0.1517757 \mathrm{E}+00$ & $0.1587633 \mathrm{E}+00$, \\
\hline $0.1659115 \mathrm{E}+00$ & $0.1732221 \mathrm{E}+00$ & $0.1806969 \mathrm{E}+00$ & $0.1883390 \mathrm{E}+00$ & $0.1961518 \mathrm{E}+00$, \\
\hline $0.2041400 \mathrm{E}+00$ & $0.2123093 \mathrm{E}+00$ & $0.2206671 \mathrm{E}+00$ & $0.2292222 \mathrm{E}+00$ & $0.2379856 \mathrm{E}+00$, \\
\hline $0.2469709 \mathrm{E}+00$, & $0.2561942 \mathrm{E}+00$ & $0.2656752 \mathrm{E}+00$ & $0.2754372 \mathrm{E}+00$ & $0.2855080 \mathrm{E}+00$, \\
\hline $0.2959203 \mathrm{E}+00$ & $0.3067128 \mathrm{E}+00$ & $0.3179307 \mathrm{E}+00$ & $0.3296266 \mathrm{E}+00$ & $0.3418615 \mathrm{E}+00$, \\
\hline $0.3547061 \mathrm{E}+00$ & $0.3682416 \mathrm{E}+00$ & $0.3825613 \mathrm{E}+00$ & $0.3977717 \mathrm{E}+00$ & $0.4139944 \mathrm{E}+00$, \\
\hline $0.4313675 \mathrm{E}+00$ & $0.4500474 \mathrm{E}+00$ & $0.4702109 \mathrm{E}+00$ & $0.4920571 \mathrm{E}+00$ & $0.5158098 \mathrm{E}+00$, \\
\hline $0.5417201 \mathrm{E}+00$ & $0.5700686 \mathrm{E}+00$ & $0.6011688 \mathrm{E}+00$ & $0.6353697 \mathrm{E}+00$ & $0.6730590 \mathrm{E}+00$ \\
\hline $\begin{array}{l}0.7146671 \mathrm{E}+00 \\
0.1000000 \mathrm{E}+01\end{array}$ & $0.7606701 \mathrm{E}+00$ & $0.8115944 \mathrm{E}+00$ & $0.8680208 \mathrm{E}+00$ & $0.9305884 \mathrm{E}+00$ \\
\hline \multicolumn{5}{|l|}{ eta $a_{-}$o $=$} \\
\hline $0.1176471 \mathrm{E}-03$ & $0.4313726 \mathrm{E}-03$ & $0.9019608 \mathrm{E}-03$ & $0.1529412 \mathrm{E}-02$ & $0.2313725 \mathrm{E}-02$, \\
\hline $0.3254902 \mathrm{E}-02$, & $0.4352941 \mathrm{E}-02$ & $0.5607843 \mathrm{E}-02$ & $0.7019607 \mathrm{E}-02$ & $0.8588235 \mathrm{E}-02$ \\
\hline $0.1031373 E-01$ & $0.1219608 \mathrm{E}-01$ & $0.1423529 \mathrm{E}-01$ & $0.1643137 \mathrm{E}-01$ & $0.1878431 \mathrm{E}-01$ \\
\hline $0.2129412 \mathrm{E}-01$, & $0.2396078 \mathrm{E}-01$ & $0.2678431 \mathrm{E}-01$ & $0.2976470 \mathrm{E}-01$ & $0.3290196 \mathrm{E}-01$ \\
\hline $0.3619608 \mathrm{E}-01$ & $0.3964706 \mathrm{E}-01$ & $0.4325490 \mathrm{E}-01$ & $0.4701960 \mathrm{E}-01$ & $0.5094118 \mathrm{E}-01$ \\
\hline $0.5501961 \mathrm{E}-01$ & $0.5925490 \mathrm{E}-01$ & $0.6364705 \mathrm{E}-01$ & $0.6819607 \mathrm{E}-01$ & $0.7290196 \mathrm{E}-01$ \\
\hline $0.7776470 \mathrm{E}-01$ & $0.8278431 \mathrm{E}-01$ & $0.8796078 \mathrm{E}-01$ & $0.9329412 \mathrm{E}-01$ & $0.9878433 \mathrm{E}-01$ \\
\hline $0.1044314 \mathrm{E}+00$ & $0.1102354 \mathrm{E}+00$ & $0.1161964 \mathrm{E}+00$ & $0.1223144 \mathrm{E}+00$ & $0.1285897 \mathrm{E}+00$ \\
\hline $0.1350224 \mathrm{E}+00$ & $0.1416128 \mathrm{E}+00$ & $0.1483616 \mathrm{E}+00$ & $0.1552695 \mathrm{E}+00$ & $0.1623374 \mathrm{E}+00$ \\
\hline $0.1695668 \mathrm{E}+00$ & $0.1769595 \mathrm{E}+00$ & $0.1845180 \mathrm{E}+00$ & $0.1922454 \mathrm{E}+00$ & $0.2001459 \mathrm{E}+00$ \\
\hline $0.2082247 \mathrm{E}+00$ & $0.2164882 \mathrm{E}+00$ & $0.2249446 \mathrm{E}+00$ & $0.2336039 \mathrm{E}+00$ & $0.2424783 \mathrm{E}+00$ \\
\hline $0.2515826 \mathrm{E}+00$ & $0.2609347 \mathrm{E}+00$ & $0.2705562 \mathrm{E}+00$ & $0.2804726 \mathrm{E}+00$ & $0.2907141 \mathrm{E}+00$ \\
\hline $0.3013166 \mathrm{E}+00$ & $0.3123218 \mathrm{E}+00$ & $0.3237787 \mathrm{E}+00$ & $0.3357441 \mathrm{E}+00$ & $0.3482838 \mathrm{E}+00$ \\
\hline $0.3614739 E+00$ & $0.3754014 \mathrm{E}+00$ & $0.3901665 \mathrm{E}+00$ & $0.4058831 \mathrm{E}+00$ & $0.4226810 \mathrm{E}+00$ \\
\hline $0.4407075 \mathrm{E}+00$ & $0.4601292 \mathrm{E}+00$ & $0.4811340 \mathrm{E}+00$ & $0.5039334 \mathrm{E}+00$ & $0.5287649 \mathrm{E}+00$ \\
\hline
\end{tabular}



$0.5558944 \mathrm{E}+00$,
$0.5856187 \mathrm{E}+00$
$0.6182693 \mathrm{E}+00$,
$0.6542144 \mathrm{E}+00$,
$0.6938630 \mathrm{E}+00$
$0.7376686 \mathrm{E}+00$,
$0.7861323 \mathrm{E}+00$,
$0.8398075 \mathrm{E}+00$,
$0.8993046 \mathrm{E}+00$,
$0.9652942 \mathrm{E}+00$,

\section{Level set $\mathbf{L 7 0}\left(50_{\mathrm{t}}, 20_{\mathrm{s}}\right)_{80}$}

\begin{tabular}{|c|c|c|c|c|}
\hline \multicolumn{5}{|l|}{ \&VERTLEVS } \\
\hline$z_{-} t o p_{-} o f_{-} n$ & $\mathrm{del}=80$ & & & \\
\hline \multicolumn{5}{|c|}{$\begin{array}{l}\text { first_constant_r } \text { rho_level }_{-} \text {leve } \\
\text { eta_theta }\end{array}$} \\
\hline .0000000 & .0002500 & .0006667 & 0012500 , & .0020000 \\
\hline .0029167, & .0040000 , & .0052500, & .0066667 , & .0082500 \\
\hline .0100000 , & .0119167 & .0140000 & .0162500 , & .0186667 \\
\hline .0212500 , & .0240000 & .0269167 , & .0300000 , & .0332500 \\
\hline .0366667 , & .0402500 & .0440000 & .0479167 , & .0520000 \\
\hline .0562500 , & .0606667 & .0652500 & .0700000 , & .0749167 \\
\hline .0800000 & .0852500 & .0906668 & .0962505 , & .1020017 \\
\hline .1079213, & .1140113 & .1202745 & .1267154 , & .1333406 \\
\hline .1401592 , & .1471838 & .1544313 & .1619238 , & .1696895 \\
\hline .1777643 & .1861929 & .1950307 & .2043451 , & .2142178 \\
\hline .2247466 & .2360480 & .2482597 & .2615432, & .2760868 \\
\hline .2921094 , & .3098631 & .3296378 & .3517651 , & 222 \\
\hline .4046373 , & .4362943 & .4721379 & .5127798 , & .5589045 \\
\hline .6112759 , & .6707432 & .7382500 & .8148403 , & .9016668 \\
\hline 1.0000000 & & & & \\
\hline etta_rho= & & & & \\
\hline .0001250, & .0004583, & .0009583 & .0016250 , & .0024583 \\
\hline .0034583, & .0046250 , & .0059583 & .0074583 , & .0091250 \\
\hline .0109583 , & .0129583, & .0151250 & 0174583 , & .0199583 \\
\hline .0226250 , & .0254583, & .0284583 & .0316250 , & .0349583 \\
\hline .0384583, & .0421250, & .0459583 & .0499583 , & .0541250 \\
\hline .0584584, & .0629583, & .0676250 & .0724583 , & .0774583 \\
\hline .0826250 , & .0879584, & .0934586 & .0991261 , & .1049615 \\
\hline .1109663, & .1171429 , & .1234950 & .1300280 , & .1367499 \\
\hline .1436715 , & .1508076 & .1581776 & .1658067 , & .1737269 \\
\hline .1819786 , & .1906118, & .1996879 & 2092815 , & .2194822 \\
\hline .2303973 , & .2421538 & .2549014 & 2688150 , & .2840981 \\
\hline .3009862 & .3197505 , & .3407014 & .3641936 , & .3906297 \\
\hline .4204658 & .4542161 & .4924589 & .5358422 , & .5850902 \\
\hline .6410096 & .7044966 , & .7765451 & .8582535 , & .9508334 \\
\hline
\end{tabular}

\section{Level set L63(50 $\left(13_{\mathrm{s}}\right)_{40}$}

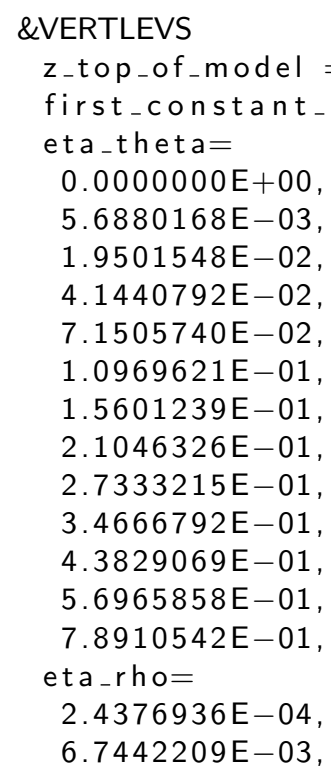

1.3001683E-03,

$1.0238313 \mathrm{E}-02$,

2.7302168E-02,

5.2491732E-02,

8.5806817E-02,

$1.2724760 \mathrm{E}-01$,

1.7681430E-01,

2.3455390E-01,

3. $0116495 \mathrm{E}-01$,

3.8034007E-01,

4.8414484E-01,

$6.4284474 \mathrm{E}-01$,

9. $2074203 \mathrm{E}-01$,

1.8688334E-03,

1. $1619608 \mathrm{E}-02$,
2.4376935E-03, 1.3001097E-02, 3. $1690016 \mathrm{E}-02$, 5.8504645E-02, 9.3444988E-02, 1.3651083E-01, 1.8770337E-01, 2. $4711467 \mathrm{E}-01$, 3. $1577647 \mathrm{E}-01$, 3. $9850461 \mathrm{E}-01$, 5. $1004976 \mathrm{E}-01$, $6.8599641 \mathrm{E}-01$, $1.0000000 \mathrm{E}+00$,

$3.1690018 \mathrm{E}-03$, 1.4544840E-02,
3. $9003098 \mathrm{E}-03$, $1.6088778 \mathrm{E}-02$, 3. $6402956 \mathrm{E}-02$, 6. $4842649 \mathrm{E}-02$, $1.0140806 \mathrm{E}-01$, 1.4609917E-01, 1.9891910E-01, $2.6003483 \mathrm{E}-01$, 3. $3092082 \mathrm{E}-01$, 4.1775790E-01, 5.3841203E-01, 7.3447162E-01,

4.7940658E-03, 1.7795164E-02, 
illustrate in this section. In Table 4 we illustrate an example of the systems settings that vary between deterministic global NWP and climate research configurations of GA7.0/G7.0. Note that this is just a description of the scientific differences rather than a full list of technical options/namelist entries that would need to be changed to apply these changes.

\begin{tabular}{lrr}
\hline $\begin{array}{l}\text { Area of the model } \\
\text { Scientific option }\end{array}$ & NWP & Climate \\
\hline Time dependent options & Gregorian & 360 day* \\
Calendar type & Non-reproducible & Double-double precision \\
Method for calculating global sums & & \\
$\quad$ Summation type & Off & On \\
$\begin{array}{l}\text { Definition of land/sea } \\
\text { Use of "coastal tiling" }\end{array}$ & Off & On \\
$\begin{array}{l}\text { Global conservation options } \\
\text { Conservative moisture/tracer advection }\end{array}$ & Off & On \\
Enforced energy conservation & & Prognostic \\
$\begin{array}{l}\text { Aerosol modelling } \\
\text { Prognostic/climatological aerosol }\end{array}$ & Climatological** & \\
\hline
\end{tabular}

Table 4: A descriptive example of GA7.0 settings that might vary with system/application. *Note that the 360 day calendar is not compulsory for GA7.0 climate jobs, but is still used in most standard jobs. ${ }^{* *}$ As discussed in the main paper, the aerosol definition is dependent on the system. Most climate runs use full UKCA Glomap-mode prognostic aerosols with with prescribed gas phase precursors. Runs on Seasonal timescales or shorter use CLASSIC aerosol climatologies, although in time we would like to replace these with Glomap-mode climatologies. Finally, we now usually include prognostic mineral dust forecasting and assimilation on NWP timescales. 